EDITORIAL

\title{
After a STEMI, is Less Sugar more Protective to Myocardium?
}

\author{
Rafael Willain Lopes ${ }^{\circledR}$ and Alexandre Hohl ${ }^{\circledR}$ \\ Hospital do Coração, São Paulo, SP - Brazil. \\ Universidade Federal de Santa Catarina, Florianópolis, SC - Brazil. \\ Editorial referring to the article: Does Tight Glucose Control During the First 24 hours of Hospitalization Reduce Scintigraphic Infarct Size in STEMI Patients?
}

The infarct size reduction in acute myocardial infarction (AMI) has been studied for more than 50 years. Animal experiments were conducted in the 1970s, followed by the first clinical studies to reduce infarct size (IS), with fibrinolytic agents or with mechanical coronary angioplasty (PTCA). The clinical experience of coronary reperfusion indicated that left ventricle function was not normalized in $30 \%$ of the patients, and despite unblocking the epicardial vessel, demonstrated hemodynamically, there was no equivalent in myocardial perfusion. New concepts emerged such as reperfusion injury, microvascular dysfunction, "noreflow" phenomenon, and stunned and hibernating myocardium, which became the focus of basic research and clinical investigation. To improve characterization, different technologies were used, such as contrast echocardiography, isotopic studies, including positron emission tomography, and magnetic resonance. ${ }^{1}$

Cardioprotection $(\mathrm{CP})$ aims to reduce IS and improve clinical outcomes. The translation of $\mathrm{CP}$ from preclinical and promising proof-of-concept studies to clinical benefit (CB) for patients has been quite unsatisfactory. Almost all these studies that did not translate into $C B$ had infarct size reduction as the primary endpoint and used protocols selected to achieve IS reduction. ${ }^{2}$

In 2001, a pioneering randomized clinical trial (RCT) conducted in Leuven, Belgium, found clear benefits in treating hyperglycemia, supporting a

\section{Keywords}

ST Elevation Myocardial Infarction; Fibrinolytic Agents; Angioplasty; Percutaneous Coronary Intervention; Myocardial Reperfusion; Ventricular Dysfunction Left; Myocardial Stunning; Diagnostic, Imaging.

\section{Mailing Address: Rafael Lopes}

Rua Desembargador Eliseu Guilherme, 147. Postal Code: 04004-030 São Paulo, SP - Brazil.

E-mail: rafanuclear@hotmail.com

DOI: https://doi.org/10.36660/ijcs.20200173 potential causal relationship between hyperglycemia and outcomes. The researchers studied 1548 critically ill patients admitted to a predominantly surgical intensive care unit (ICU), maintaining healthy fasting blood glucose concentrations (80-110 mg/dL), which led to a reduction in morbimortality, compared with tolerance to hyperglycemia up to the renal threshold $(215 \mathrm{mg} / \mathrm{dL}){ }^{3}$

Later, the Leuven's research group confirmed the clinical benefit in critically ill adults admitted to a clinical ICU $(\mathrm{n}=1200)$ and severely ill children $(\mathrm{n}=$ 700). ${ }^{4,5}$ Subsequent mechanistic studies attributed the benefit obtained by rigid glucose control to a protection against glucose toxicity and not to glucoseindependent insulin effects. ${ }^{6,7}$

Despite the promising effects of rigid glucose control in the first controlled studies, the benefit was not confirmed in subsequent multicenter studies and the NICE-SUGAR study found potential damage. ${ }^{7-10}$ The increased risk of mortality in NICE-SUGAR was subsequently attributed to increased incidence of hypoglycemia. ${ }^{11}$ This discrepancy can be explained by methodological differences between trials, and not by a different combination of cases.

Hyperglycemia (HGL) in the setting of myocardial revascularization (MR) is associated with increased adverse effects in patients with and without diabetes. Data suggest that acute HGL peri-procedure causes increased inflammation, platelet activity, endothelial dysfunction, and is associated with plaque instability and IS. While peri-procedure glycemia is an independent predictor of adverse effects in patients undergoing MR, treatment strategies remain uncertain. ${ }^{12}$

It is also known that cardioprotective actions of ischemic postconditioning (PCISQ) against ischemia/ reperfusion injury (I/LR) are abolished in diabetic hearts. Several drugs used for treating diabetes have recently shown a reduction in difficult cardiovascular outcomes. Animal studies have sought to investigate the combined effect of PCISQ and these drugs on myocardial function and IS. ${ }^{13}$ 
The scintigraphy image with a single photon emission computed tomography (SPECT) technique with sestamibi-99mTc (technetium) is based on the integration of myocardial perfusion and myocyte integrity. Sestamibi is distributed in the myocardium in proportion to blood flow ${ }^{14}$ and cardiac uptake is dependent on a normal mitochondrial function. ${ }^{15}$

In the early 2000s Gibbons et al. ${ }^{16}$ had already demonstrated in 2 publications with a wide literature review that SPECT imaging with sestamibi-99mTc was the best available tool to evaluate IS and with potential to serve as a surrogate outcome to discover advantages of new therapies that could be equivalent to those existing in relation to early mortality, ${ }^{16}$ as well as to evaluate possible incremental benefits in multicenter studies. ${ }^{17}$

In 2011 Gibbons, ${ }^{18}$ in an excellent review article, highlighted that SPECT with sestamibi-99mTc had multiple evidences that validated its clinical usefulness, being used as surrogate outcome in several randomized trials, and in some of them, both early and later imaging were used to evaluate myocardium at risk and myocardial salvage. The author recognized that despite its limitations, SPECT is a well-validated measure. ${ }^{18}$

In this edition of the IJCS, the authors Gulsen et al. ${ }^{19}$ integrating this knowledge sought to investigate whether rigid glycemic control in the first 24 hours after AMI with ST elevation (STEMI) may have a cardioprotective role using IS through SPECT as an outcome, searching for a possible incremental benefit of this strategy, as already largely supported in the literature, and should be congratulated by this design based on robust literature, both for the intervention tested and the method used as a surrogate outcome to evaluate the response. The limitations of the singlecenter study stand out and, in relation to the evaluation of the benefits of a new therapeutic approach, despite the technical and logistical difficulties, the importance of determining myocardium at risk and the effectively spared seem relevant for the analysis of the results, whether positive or negative.

Despite promising experimental studies and proofof-concept clinical studies such as these, interventions seeking to limit IS failed to improve clinical outcomes in STEMI. Although IS alone has prognostic value, Bochaton et al., ${ }^{20}$ demonstrated that other variables in STEMI treated with angioplasty may be associated with clinical outcomes, regardless of IS. Among these variables are risk factors, comorbidities, post-treatment variables, and simultaneous treatments. ${ }^{20}$

Therefore, $\mathrm{CP}$ should not only focus on the reduction of IS, but also on several factors that should contribute to clinical outcomes in the short and long term. ${ }^{2}$ In this context, studies such as this seek to shed light on new approaches to known factors, in the treatment of

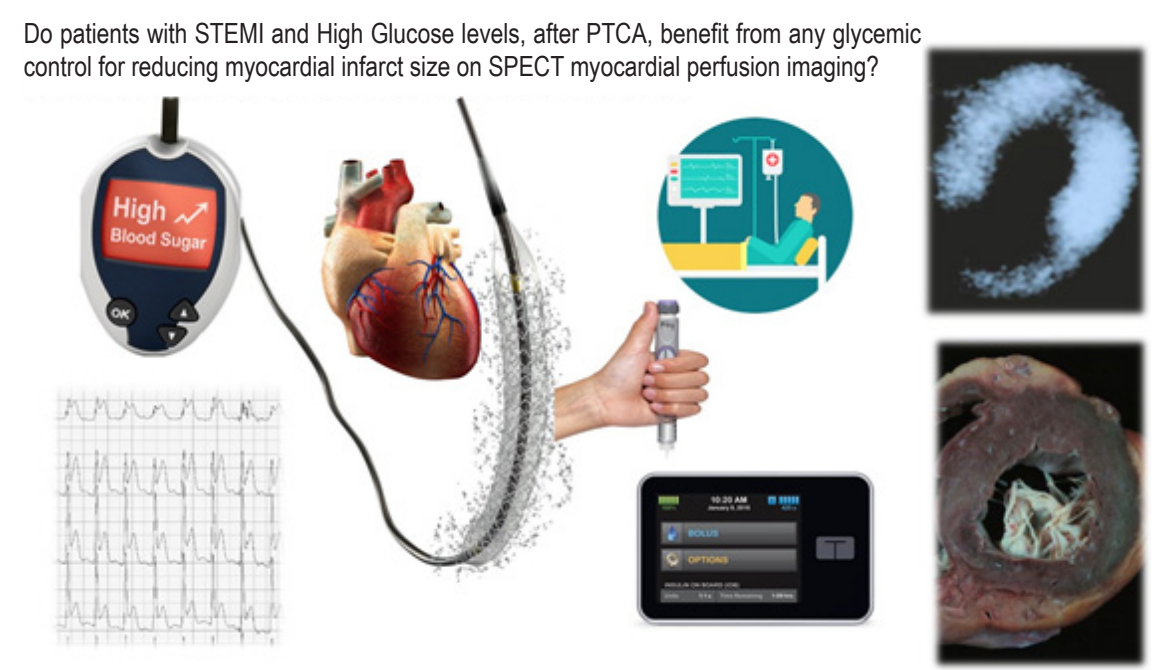

Figure 1 - The image illustrates the idea of high glucose levels as a risk factor that could implicate In the outcomes of STEMI patients and when controlled immediately after the PTCA could reduce the infarct size evaluated by myocardial perfusion spect, as surrogate outcome. STEMI ST Elevation Myocardial Infarction PTCA Percutaneous Transluminal Coronary Angioplasty. 
AMI, such as glycemic levels, trying to answer whether after STEMI, less sugar means more protection for the myocardium, aiming to improve outcomes of this pathology that brings such a high cost for the patient and society. However, strict glucose control remains

\section{References}

1. Ferreira R. The reduction of infarct size--forty years of research. Rev Port Cardiol. 2010;29(6):1037-53.

2. Heusch G. Coronary microvascular obstruction: the new frontier in cardioprotection. Basic Res Cardiol. 2019;114(6):45.

3. Van den Berghe G, Wouters P, Weekers F, Verwaest C, Bruyninckx F, Schetz $\mathrm{M}$, et al. Intensive insulin therapy in critically ill patients. N Engl J Med. 2001;345(19):1359-67.

4. Van den Berghe G, Wilmer A, Hermans G, Meerssemann W, Wouters PJ, Milants I, et al. Intensive insulin therapy in the medical ICU. N Engl J Med. 2006;354(5):449-61.

5. Vlasselaers D, Milants I, Desmet L, Wouters PJ, Vanhorebeek I, van den Heuvel I, et al. Intensive insulin therapy for patients in paediatric intensive care: a prospective, randomised controlled study. Lancet. 2009;373(9663):547-56.

6. Marfella R, Filippo C, Portoghese M, Ferraraccio F, Rizzo MR, Siniscalchi $\mathrm{M}$, et al. Tight glycemic control reduces heart inflammation and remodeling during acute myocardial infarction in hyperglycemic patients. J Am Coll Cardiol. 2009;53(16):1425-36.

7. Goldberg PA, Siegel MD, Sherwin RS, Halickman JI, Lee M, Bailey VA, et al Implementation of a safe and effective insulin infusion protocol in a medical intensive care unit. Diabetes Care. 2004;27(2):461-7.

8. Preiser JC, Devos P, Ruiz-Santana S, Mélot C, Annane D, Groeneveld J, et al. A prospective randomised multi-centre controlled trial on tight glucose control by intensive insulin therapy in adult intensive care units: the Glucontrol study. Intensive Care Med. 2009;35(10):1738-48.

9. Brunkhorst FM, Engel C, Bloos F, Meier-Hellmann A, Ragaller M, Weiller N, et al. Intensive insulin therapy and pentastarch resuscitation in severe sepsis. N Engl J Med. 2008;358(2):125-39.

10. Finfer S, Blair D, Bellomo R, Yu-Shwo Su S, Foster D, Dhingra V, et al. Intensive versus conventional glucose control in critically ill patients. N Engl J Med. 2009;360(13):1283-97.

11. NICE-SUGAR Study Investigators, Finfer S, Liu B, Chittock DR, Norton R, Myburgh JA, et al. Hypoglycemia and risk of death in critically ill patients. N Engl J Med. 2012;367(12):1108-18. highly debated, which leads to wide variations in practice $^{21}$ and leaves us a reflection, paraphrasing Alan Turing, British scientist considered the father of computing: "We can only see a little of the future, but it is enough to realize that there is much to do".

12. Ujueta F, Weiss EN, Sedlis SP, Shah B. Glycemic control in coronary revascularization. Curr Treat Options Cardiovasc Med. 2016;18(2):12.

13. Bayrami G, Karimi P, Agha-Rosseini F, Feyzizadeh S, Badalzadeh R. Effect of ischemic postconditioning on myocardial function and infarct size following reperfusion injury in diabetic rats pretreated with vildagliptin. J Cardiovasc Pharmacol Ther. 2018;23(2):174-83.

14. Okada RD, Glover D, Gaffney T, Williams S. Myocardial kinetics of technetium-99m-hexakis-2-methoxy-2-methylpropyl-isonitrile. Circulation. 1988;77(2):491-8.

15. Crane P, Laliberté R, Heminway S, Thoolen M, Orlandi C. Effect of mitochondrial viability and metabolism on technetium-99msestamibi myocardial retention. Eur J Nucl Med. 1993;20(1):20-5.

16. Gibbons RJ, Miller TD, Christian TF. Infarct size measured by single photon emission computed tomographic imaging with $(99 \mathrm{~m}) \mathrm{Tc}-$ sestamibi: a measure of the efficacy of therapy in acute myocardial infarction. Circulation. 2000;101(1):101-8.

17. Gibbons RJ, Valeti US, Araoz PA, Jaffe AS. The quantification of infarct size. J Am Coll Cardiol. 2004;44(8):1533-42.

18. Gibbons RJ. Tc-99m SPECT sestamibi for the measurement of infarct size. J Cardiovasc Pharmacol Ther. 2011;16(3-4):321-31.

19. Gulsen K, Ayca B, Baskurt M, Okcun B, Ersanli KM. Does Tight Glucose Control During the First 24 hours of Hospitalization Reduce Scintigraphic Infarct Size in STEMI Patients?. Int. J. Cardiovasc. Sci. [Internet]. [cited 2020 Sep 09]. Available from: http://www.scielo.br/scielo.php?script=sci_arttext\&pid=S235956472020005007203\&lng=en. In press 2020. Epub Aug 07, 2020. http://dx.doi.org/10.36660/ijcs.20200020.

20. Bochaton T, Claeys MJ, Garcia-Dorado D, Mewton N, Bergerot C, Jossan C, et al. Importance of infarct size versus other variables for clinical outcomes after PPCI in STEMI patients. Basic Res Cardiol. 2019;115(1):4.

21. Preiser JC, Straaten HMO. Glycemic control: please agree to disagree. Intensive Care Med. 2016;42(9):1482-4. 